\title{
THREE DIVORCES: A SOCIOLOGICAL SCIENTIFIC ANALYSIS
}

\section{तीन तलाक: एक समाज वैज्ञानिक विश्लेषण}

\author{
Dr. Prabha Sharma ${ }^{1} \mathbb{}$, Dinesh Sharma ${ }^{2}$ \\ ${ }^{1}$ Research Directory, Principal, Government College, Sambhal, India \\ ${ }^{2}$ Research Student Sociology, Govt. Graduate College, Rampur, Mahatma Jyotiba Phule Ruhelkhand \\ University, Bareilly, India
}

DOI: https://doi.org/10.29121/granthaalayah.v8.i10.2020.2029

Article Type: Research Article

Article Citation: Dr. Prabha

Sharma, and Dinesh Sharma. (2020).

THREE DIVORCES: A

SOCIOLOGICAL SCIENTIFIC

ANALYSIS. International Journal of

Research -GRANTHAALAYAH,

8(10), 182-186.

https://doi.org/10.29121/granthaa

layah.v8.i10.2020.2029

Received Date: 14 October 2020

Accepted Date: 31 October 2020

Keywords:

इस्लामी शरीअत में निकाह

तलाक एवं हलाला

बहु-विवाह इस्लामिक कानून

भारतीय संविधान

\section{ABSTRACT}

English: The article presented describes the marriage (nikah) divorce, multi-marriage Shariah and Indian constitution due to the religious trends of the Muslim class in Indian society. India is a sovereign, socialist, secular, democratic republic with a parliamentary system of government. In the republic of the same parliamentary system, in the present time, the right of Muslim women to be violated, in the form of divorce, in the form of divorce, and there is no more dowry, the main reason for such exploitation is lack of proper and complete knowledge of religion. , Wrong use of the rule of Sharia law is to be superstitious and orthodox and to not give legal form to marriage with very important religious marriage. The articles presented are based on Purnataya Second type of information like - Islamic book, Indian constitution, newspaper, website of Ministry of Information and Broadcasting (Government of India) etc.

Hindi: प्रस्तुत लेख में भारतीय समाज में मुस्लिम वर्ग के धार्मिक प्रवृत्तियों से हो रहे विवाह (निकाह) तलाक, बहु विवाह शरीअत एवं भारतीय संविधान का वर्णन किया गया है। भारत में संसदीय प्रणाली की सरकार वाला एक प्रभुसत्ता सम्पत्र, समाजवादी, धर्म निरपेक्ष, लोकतंत्रात्मक गणराज्य है। उसी संसदीय प्रणाली की सरकार वाले गणराज्य में वर्तमान समय मुस्लिम महिलाओं के अधिकारों का हनन कही निकाह के रूप में, कही तलाक के रूप में तो कही दहेज अधिक न मिलने के कारण इस प्रकार के शोषण का मुख्य कारण धर्म का सही और पूरा ज्ञान न होना, शरीअत कानून के नियम का गलत प्रयोग अन्धविश्वास एवं रुढ़िवादी होना एवं अत्यन्त महत्वपूर्ण धार्मिक विवाह के साथ ही साथ विवाह को कानूनी रूप प्रदान न किया जाना है। प्रस्तुत लेख पूर्णताया द्वितीय प्रकार की सूचनाओं पर आधारित हैं जैसे - इस्लामिक पुस्तक, भारतीय संविधान, समाचारपत्र, सूचना एवं प्रसारण मंत्रालय (भारत सरकार) की बेवसाइड इत्यादि।

\section{1. प्रस्तावना}

भारत एक धर्म निरपेक्ष राज्य है और यहाँ नागरिकों पर लागू करने के लिए अनेक कानून है। भारत के मुस्लिम समुदाय पर कुछ मामलों में तो देश का सामान्य कानून लागू होता है तो कुछ मसले, मुस्लिम पर्सनल कानून के आधार पर हल होते है। प्रस्तुत शोधपत्र में हम मुस्लिम औरतों के लिए बेहद महत्वपूर्ण मुस्लिम विवाह-विघटन अधिनियम 1939 और मुस्लिम महिला (विवाह-विच्छेदन) अधिनियम 1986 का विश्लेषण कर रहे हैं। 
कुरआन शरीफ में दिए गए अधिकार ही औरतों को मिल जाएं तो स्थिति में आमूलचूल बदलाव आ सकता है। क्योंकि कुरआन शरीफ जगहजगह पर लिंगीय समानता की बात करता है। कुरआन की आदर्श व्याख्या के मुताबिक महिला व पुरूष दोनों अल्लाह की संताने हैं और दोनों के समान दर्जा हासिल हैं लेकिन इनके साथ भेदभाव किया जाता है।

वर्तमान में मुस्लिम महिला (विवाह-विच्छेदन) अधिनियम 1986 के द्वारा तलाक के मामलें में अब मुस्लिम महिला की स्थिति काफी हद तक सुधर गई है, लेकिन पूर्व में प्रचलित किसी भी समय एक साथ तीन तलाक कह देने से जो तलाक माना जा रहा है यह मुस्लिम महिलाओं के प्रति अन्याय है।

भारतीय समाज में एक सोच बहुत ज्यादा प्रभावशाली है और वह है बिना सोचे समझे किसी प्रथा को मान्यता दे देना। सम्पूर्ण ज्ञान ना होते हुए भी कुछ परम्पराओं को मान देने लगते है जो आगे चलकर सभी अन्य लोगों के लिए दुखदायी हो जाता है। [1]

इसी प्रकार की सोच का परिणाम है वर्तमान में प्रचलित "तीन तलाक की परम्परा। तीन तलाक की परम्परा आज मुस्लिम समाज की महिलाओं के लिए एक अभिशाप बन गई है। वर्तमान में समाज में इस परम्परा ने ना जाने कितनी महिलाओं के जीवन को नारकीय बना दिया है और ना जाने कितने अनगिनत घर-परिवारों को नष्ट किया है वर्तमान में अब धर्म और परम्परा के नाम पर सुधार की जरूरत एवं आवश्यकता है।

आधुनिक समाज में कुरान का सहारा लेकर भी इस तीन तलाक की प्रथा का बचाव नही किया जाना चाहिए। दर असल, यह मामला पवित्र कुरान का नही बल्कि उसकी अलग-अलग व्याख्याओं का है। यहाँ यह बताना जरूरी है कि इस्लाम धर्म के सबसे पवित्र ग्रंथ कुरान में तीन तलाक का जिक्र नहीं हैं। लेकिन पुरूषवादी सोच के चलते मुस्लिम समाज में यह कुरीति प्रचलित है। तीन तलाक का वर्तमान मुद्दा धर्म की आड़ में किसी भी नागरिक के मौलिक अधिकारों का हनन अवैध है। वर्तमान में जो मुस्लिम महिलाएं लम्बे समय से इस प्रथा के खिलाफ आंदोलन चला रही है वे भी इस्लाम और कुरान में आस्था रखती है, और वे कहती है कि इस्लाम या कुरान उनके अधिकार छीनने या कम करने को नहीं कहता है। मुस्लिम पर्सनल लॉ में संशोधन से न तो कुरान की पवित्रता पर कोई आंच आएंगी, न इस्लाम का कोई नुकसान होगा। [2] 1972 में पहली बार केरल हाईकोर्ट में चीफ जस्टिस पी खालिद ट्रिपल तलाक को इस्लाम और भारतीय संविधान दोनों की मूल भावना के खिलाफ करार दियें। उसके बाद 2002 में शमीम आरा के मामले में भी सुप्रीम कोर्ट ने ट्रिपल तलाक को भारतीय संविधान और इस्लाम मूल भावना के खिलाफ बताते हुए अमान्य करार घोषित किया गया।

इसी तरह सामाजिक कुरीतियों के खिलाफ अब तक हुए ऐतिहासिक संघर्षो में 22 अगस्त 2017 का दिन एक और स्मरणीय दिन बन गया आज ही के सर्वोच्च न्यायालय ने मुस्लिम महिलाओं के लिए अभिशाप बन चुके "तीन तलाक, को असंवैधानिक करार दिया है 32 वर्ष पूर्व परिवर्तन की जो मात्रा शाहबानों के माध्यम से मुस्लिम समाज में शुरू हुई थी जिसके समक्ष कट्टरपंथी पुरानी परम्परा तथा मुस्लिम वोट बैंक की राजनीति अवरोधक बन गये थे, वह लंबित यात्रा अब सायराबानों की तरह मुस्लिम महिलाओं के माध्यम से अपने पड़ाव पर सफलपूर्वक पहुँच गई है। [3]

मुस्लिम समाज में तीन तलाक, जैसे घोर अनैतिक सामाजिक प्रथा का आखिरकार अंत हो ही गया। देश के सर्वोच्च न्यायालय ने देश की आधी आबादी के बीच समानता की एक आधारशिला रख ही है। महिलाओं के तीन तलाक से सम्बन्धित मुद्दा नये भारत के निर्माण में जहाँ यह एक बड़ा कदम है वही इस फैसले ने भविष्य को लेकर भी संकेत दे दिए हैं कि समानता, गरिमा और अधिकार की लड़ाई में धर्म और परम्परा को थोड़ा पीछे हटना ही पड़ेगा। [4]

तीन तलाक का मुद्दा शुद्ध रूप से सामाजिक विषय है पर जिस प्रकार यह ऐतिहासिक फैसला आया है इसके बाद यह जानना स्वाभाविक है कि क्या राजनीतिक इच्छा शक्ति के बिना यह सामाजिक बदलाव संभव था? जवाब नहीं, दरअसल पहली बार सरकार ने खुलकर बदलाव का नजरियां रखा था। कोर्ट को बताया गया था कि विभित्र मुस्लिम देशों में एक साथ तीन तलाक की परम्परा को ख़ारिज किया जा चुका है। [5]

मुस्लिम समाज में एक बार में तीन तलाक की प्रथा के खिलाफ लम्बी लड़ाई लड़ने वाले संगठन “भारतीय मुस्लिम महिला आन्दोलन, (सी.एम.एम.ए) ने सुप्रीम कोर्ट के फैसले का स्वागत करते हुए कहा कि अब सरकार और राजनीतिक दलों को मिलकर हिंदू विवाह अधिनियम की तर्ज पर "मुस्लिम परिवार कानून, बनाना चाहिए।

(वी.एम.एम.ए) की संस्थापक-नूरजहां सफिया नियाज ने कहा कि सुप्रीम कोर्ट के ऐतिहासिक फैसले में मुसलिमों में एक बार में तीन तलाक की प्रथा "अमान्य अवैध, और असंवैधानिक मानी गई।

शीर्ष अदालत ने $3: 2$ के मत से सुनाए गए फैसले में तीन तलाक को कुरान के मूल तत्व के खिलाफ बताया और कहा कि इस फैसले को हम सुप्रीमकोर्ट के फैसले का स्वागत करते है। यह इन सभी महिलाओं की जीत है जो इस लम्बी लड़ाई में हमारे साथ थी। [6]

वर्तमान समाज सुधार संगठनों और इसमें सक्रिय पुरूषों का मानना है कि मुसलमान औरत को कुरान में बहुत अधिकार मिलते हैं। शिक्षा के माध्यम से उसे उन अधिकारों के उपयोग के योग्य बनाना उनका मुख्य उद्देश्य था। वर्तमान सदी के आरम्भ मं० हाजी और मौलवी नजीर अहमद ने मुसलमान औरत को कुरान प्रदत्त अधिकारों के प्रयोग करने में भी शिक्षा और पर्दा की वजह से अक्षम बताया। [7]

भारत में मुसलमान स्त्तियों की स्थिति का प्रश्न स्त्रियों के कानूनी संरक्षण से भी जुड़ा है। शाहबानों केस के बाद समाज शरीयत समर्थक तथा शरीयत में संशोधन के समर्थक दो खेमों के बंट गया। चूकिं हिंदू सांप्रदायिक तत्वों को शाहबानों के बाद इस्लाम समस्त जीवन पद्धति से छेड़छाड़ करने का एक बहाना सा मिल गया उन्होंने मुसलमान स्त्री की दयनीय स्थिति पर घड़ियाली आंसू बहाकर यूनीफार्म सिविल कोड और निजी व्यक्तिगत कानूनों को समाप्त करने की जोर-शोर से वकालत करनी आरंभ कर दी। इसका उतना ही शिद्दत से रूढ़िवादी मुसलमानों ने कड़ा विरोध किया। लेकिन विभिन्न मुसलमान समाजों के प्रगतिशील, उदारवादी तत्वों ने अपना धीरज नही खोया। उन्होंने मुसलमान औरत के तलाक के अधिकार को ऐतिहासिक धार्मिक तथा अन्य इस्लामी देशों में प्रचलित कानूनों के संदर्भ में देखना प्रारम्भ कर दिया। [8]

जहाँ तक मुस्लिम महिलाओं में तलाक का सवाल है तो सन् 1939 से पूर्व मुस्लिम महिलाओं की स्थिति तलाक के मामले में बहुत ही बदतर थी। तलाक देना मुस्लिम पुरूष का एक निरंकुश अधिकार था। वह जब चाहे अपनी पत्नी को तीन शब्दों का उच्चारण कर तलाक दे सकता था। इतना ही नहीं एक मुस्लिम पुरूष तलाक के बाद केवल इद्दत की अवधि तक की पत्नी को भरण-पोषण का खर्चा देने के लिए बाध्य है इस स्थिति में महिला की स्थिति को और ज्यादा विकृत बना दिया और पति के लिए पत्नी को तलाक देना आसान होने से तलाक एक आम बात बन गई। सन् 1939 से पूर्व पत्नी 


\section{डॉ. प्रभा शर्मा, दिनेश शर्मा}

केवल पारस्परिक सहमति के आधार पर मुबारक तलाक ले सकती थी। किन्तु उसे अपनी तमाम मेहर की रकम का त्याग करना पड़ता था। इसके अलावा पति की सहमति से ही ऐसा तलाक सम्भव था। इसके अलावा इला यानि आत्मसंयम की प्रतिज्ञा, जिहार यानि पीड़क तुलना एवं लियन यानि पर पुरूष गमन के झूठे आरोप के आधार पर तलाक ले सकती थी, किन्तु इसे सिद्ध करना बहुत मुश्किल कार्य था तथा इसके लिए न्यायालय की डिग्री प्राप्त करना आवश्यक था यानि पति-पत्नी के बीच में तलाक के मामले में स्थिति बराबर नहीं थी। पहली बार सन् 1939 में इस अधिनियम द्वारा पत्नी को भी तलाक देने सम्बन्धी कुछ अधिकार भी प्राप्त है जैसे अगर पति के एक से अधिक पत्नियां है तो दोनों के साथ भेदभाव बरतने एवं असमानता का व्यवहार करने के आधार पर न्यायालय में याचिका दायर कर तलाक ले सकती है। इतना ही नहीं अब तो इला, जिहार, लियन एवं मुबारत प्रकार के तलाक को भी इस अधिनियम द्वारा मान्यता मिल चुकी है एवं मुस्लिम पति अगर धर्म परिवर्तन करता है तो परम्परा के अनुसार स्वतः ही पति-पत्नी के बीच तलाक हो जायेगा। इस अधिनियम द्वारा तलाक के मामले में अब मुस्लिम महिलाओं की स्थिति काफी हद तक सुधर गई है। फिर भी जैसा कि हम जानते है कि एक मुसलमान व्यक्ति एक ही समय में चार पत्नियां रख सकता है और उसे जब चाहे तलाक दे सकता है। [9]

अतः आज भी पति एवं पत्नी के बीच भयंकर असमानता मौजूद है। लेकिन वर्तमान समय में राज्य सेवा नियम लागू है जो भारत में सभी जाति के लोगों पर समान रूप से प्रभाव रखता है। वर्तमान में सरकारी कर्मचारी या कोई भी व्यक्ति जो सरकारी सेवारत है एक समय में एक ही पत्नी रख सकता है चाहे वह हिन्दू हो अथवा मुसलमान अथवा अन्य जाति का। इस प्रकार जहाँ तक सरकारी कर्मचारियों का सवाल है मुसलमान होते हुए भी एक से ज्यादा पत्नी रखने पर अंकुश लगा हुआ है। जहां तक पति-पत्नी के बीच पुनर्विवाह का सवाल है तो मुस्लिम विधि इतनी सरल नहीं है जितनी कि हिंदू विधि क्योंकि हिन्दू विधि में धारा 15 के तहत आसानी से पुनर्विवाह हो जाता है जबकि मुसलमान विधि के अनुसार तलाकशुदा स्त्री का विवाह तलाक के बाद अन्य व्यक्ति के साथ होना चाहिए फिर दोनों के बीच लैंगिक सम्बन्धों की स्थापना होनी चाहिए उसके बाद दोनों के बीच तलाक होने के पश्चात ही पुनः विवाह पुराने पति-पत्नी के बीच हो सकता है अन्यथा नहीं और अगर बीच का विवाह नही होता तो तलाक के बाद उन्हीं पति-पत्नी के बीच का विवाह शून्य होगा।

इस स्थिति की वजह से अवश्य ही मुस्लिम पति पर तलाक लेने के मामले में भी अंकुश लगा है क्योंकि एक बार गलती से भी तलाक दे देने की अवस्था में बीच में विवाह की शर्त जरूरी है अतः ऐसा प्रावधान स्वतः ही तलाक पर रोक का कार्य करता है जो शर्त हिन्दू विधि में लागू नहीं है किन्तु हिन्दू विधि में तो तलाक स्वयं ही अपने आप में एक जटिल प्रक्रिया है। [10]

तलाक-ए-विद्धत अर्थात् तीन तलाक पर शीर्ष अदालत की 5 सदस्यीय खंडपीठ का निर्णय बहुमत से है। इस फैसले के अनुसार तलाक की यह प्रक्रिया संविधान में दिए गए समानता के अधिकार का हनन है और यह संविधान का हिस्सा नहीं है, इसलिए इसे संविधान में दी गई यह मजहवी आजादी (अनु 25) में संरक्षण नहीं मिल सकता। इसके लिए बहुमत के आधार पर शरीयत कानून 1937 की धारा -2 में इस तलाक को दी गयी मान्यता को भी निरस्त कर दिया जजों की खंडपीठ में न्यायाधीश नरीमन न्यायाधीश ललित और न्यायाधीश कुरियन ने "तीन तलाक को असंवैधानिक बताते हुए मुस्लिम महिलाओं के अधिकारों पर आघात करने वाला बताया, वही मुख्य न्यायाधीश खेहर और न्यायाधीश नजीर इसके पक्ष में नजर आये। तलाक-ए विद्दत सुत्री समुदाय में गत 1000 वर्षों से चला आ रहा है और इसकी स्वीकृत पुरूषों को दी गई है। [11]

इस ऐतिहासिक निर्णय का परिणाम यह होगा कि अब भारत में कोई भी शौहर अपनी बेगम को मनमाने ढंग से तलाक-तलाक-तलाक कहकर नही छोड़े तो महिलाओं के मन में तलाक को लेकर बैठा भय खत्म होगा। अदालत में इस क्रांतिकारी फैसले की नींव उन मुस्लिम महिलाओं के लिए जिन्होंने इस्लामी कट्रपंथियों की परवाह किये बिना न्यायालय का दरवाजा खटखटाया। इन महिलाओं को उनके पास कोरियर, स्पीडपोस्ट, चिट्ठी-तार, क्हाट्सएप, फोन के माध्यम से या फिर अखबार में विज्ञापन देकर और लड़की को बिना कारण दिये तलाक दे दिया था। [12]

सायराबानों, इशरतजहां और जाकिया सोनम ऐसी महिलाएं है जिन्होंने अपने साहस के बल पर मुस्लिम समाज में व्याप्त सामाजिक कलंको पर विमर्श को एक नई दिशा दी है। उत्तराखण्ड में काशीपुर निवासी सायराबानों के शौहर ने डेढ़ दशक तक शादी के बंधन में रहने के बाद वर्ष 2015 में तलाक दे दिया था। सायरा ने 2016 में शीर्ष अदालत में याचिका दाखिल कर तलाक-ए-विद्दत (तीन तलाक) बहु विवाह और निकाह हलाला को संविधान के मौलिक अधिकारों के हनन को गैर-कानूनी घोषित करने की मांग उठायी। इस तरह प० बंगाल में हावड़ा की इशरतजहाँ ने अगस्त 2016 में सर्वोच्च न्यायालय में इस सामाजिक कलंक तीन तलाक के विरूद्ध याचिका दाखिल की थी। उनके पति ने दुबई से फोन पर तीन बार तलाकतलाक-तलाक कह कर रिश्ता खत्म कर दिया था। वहीं भारतीय मुस्लिम महिला आंदोलन का नेतृत्व करने वाली जाकिया ने मुस्लिम समाज में तीन तलाक एवं अन्य कुरीतियों के विरूद्ध हस्ताक्षर अभियान चलाया था जिसमें हजारों मुस्लिम महिलाओं ने बढ़-चढ़कर भाग लिया। [13]

सुप्रीम कोर्ट के निर्णय के बाद केन्द्र सरकार मुस्लिम महिला (विवाह अधिकार संरक्षण) अध्यादेश 2018 जिसे 19 सितम्बर 2018 को जारी किया जिसे राष्ट्रपति ने मंजूरी दी। यह अध्यादेश तलाक कहने को, जिसमें लिखित और इलेक्ट्रानिक दोनों रूप शामिल हैं इसे कानूनी रूप अमान्य एवं गैर कानूनी बनाया गया। इस अध्यादेश में तीन तलाक देने पर पति को तीन साल सजा का प्रावधान है। [14]

तीन तलाक को सर्वोच्च न्यायालय द्वारा अमान्य घोषित होने के बाद सरकार द्वारा तीन तलाक बिल संसद के दोनों सदनों - 25 जुलाई 2019 को लोकसभा में तथा 30 जुलाई 2019 को राज्यसभा में पास हुआ। 1 अगस्त 2019 को राष्ट्रपति की मंजूरी के बाद तीन तलाक कानून बन गया। इस कानून को 19 सितम्बर 2018 से लागू माना गया है। [15]

तीन तलाक विधेयक के प्रमुख प्रावधान -

1) तलाक-ए-बिद्दत यानी तुरन्त तीन तलाक को रद्द, इसे गैर कानूनी बनाना।

2) तलाक-ए-बिद्दत को संज्रेय अपराध बनाना।

3) तीन साल की सजा का प्रावधान।

4) पीड़ित महिला पति से गुजारा भत्ता की मांग कर सकती है।

5) पीड़ित महिला अगर अनुरोध करती है तो मजिस्ट्रेट समझौते की अनुमति दे सकता है।

6) पीड़ित महिला नाबालिग बच्चों को अपने साथ रख सकती है या नहीं इसके बारे में मजिस्ट्रेट तय करेगा।

International Journal of Research -GRANTHAALAYAH 
यह अध्यादेश विवाहित मुस्लिम महिला के अधिकारों की सुरक्षा उनके पति द्वारा तात्कालिक एवं अपरिवर्तनीय, तलाक-ए-बिद्दत के प्रचलन को रोंकेगा। तलाक से पीड़ित महिला को आजीविका भत्ता एवं नाबालिक बच्चों के संरक्षण का अधिकार प्रदान करेगा।

प्रस्तुत शोधपत्र में मुख्य रूप से इस्लाम में स्ती को प्राप्त अधिकारों तथा उनसे जुड़ा तीन तलाक के गम्भीरमुद्दे पर प्रकाश डालते हुए तीन तलाक के मुद्दे का समाज वैज्ञानिक विश्लेषण किया गया है। आज बदलते भारतीय मुस्लिम समाज में महिलायें तलाक की अपनी पुरानी विचार धाराओं एवं परम्पराओं को पीछे छोड़ने का प्रयास कर रही है। आज शोषण सह रही औरतों ने अब लामबंद होना शुरू कर दिया है। वे अब मजहब के नाम पर किए जाने वाले किसी भी जुल्म को रहने को तैयार नहीं है। यह बात औरतों ने समझ ली है और मजहब के नाम पर सभी हदबंदियों को तोड़ना शुरू कर दिया है। कोई भी परम्परा जो मानवता के खिलाफ हो, इंसान की अस्मिता के खिलाफ हो, वह कितनी भी पुरानी और स्थायी क्यों न हो उसका जमकर विरोध होना चाहिए, उसे उखाड़ फेंकना चाहिए। किसी सभ्य समाज की बुनियाद अमानवीय परंपराओं पर नही रखी जा सकती।

उपरोक्त सभी तथ्यों को संज्ञान में रखते हुए तीन तलाक की समस्या से मुक्ति हेतु निम्न सुझाव प्रस्तुत किये जा सकते है:-

1) तीन तलाक गैर कानूनी है, हलाला की घटनाएं इसी से जुड़ी है, अतः इसके लिए तुरन्त कठोर कानून अपनाया जाना चाहिए।

2) वर्तमान में अगर तीन तलाक बंद होगा तो हलाला भी बंद होगा, हलाला जो मुस्लिम महिलाओं के जीवन का शर्मनाक हिस्सा है, के लिए फास्ट ट्रैक कोर्ट बनना चाहिए।

3) 1939 का मुस्लिम विवाह विच्छेद कानून मुसलमान औरत का तलाक के लिए कोर्ट जाने की इजाजत तो देता है लेकिन मुस्लिम मर्द द्वारा दिये जाने वाले तीन तलाक पर रोक नहीं लगाता इस कानून में भी बदलाव की सखत्त जरूरत एवं वर्तमान आवश्यक है।

4) महिलाओं के सामाजिक विकास हेतु विकसित कानूनों को अमल में लाना।

5) भेदभाव और हिंसा पर आधारित सभी धार्मिक और परम्परागत प्रथाओं को अपराध अधिनियम के दायरे में लाना चाहिए।

6) एक से अधिक विवाह की इजाजत में सुधार की आवश्यकता है। पहले वाली पत्रियों के लिए तलाक का विकल्प शामिल होना चाहिए।

7) गुजारा भत्ता और उत्तराधिकार के संबंध में विस्तृत एवं पर्याप्त अधिकार व्यवहारिक तौर पर मुस्लिम महिलाओं को मिलने चाहिए।

8) तीन तलाक असंवैधानिक घोषित होने के बाद भी जो लोग इसका इस्तेमाल कर रहे है इसके लिए सख्त कानून/दण्ड होना चाहिए जिससे लोगों में भय व्याप्त हो और वे इसका दुरूपयोग करने से बचें।

9) सबसे बड़ी आवश्यकता मुस्लिम महिलाओं के स्वयं जागरूक होने की है क्योंकि चन्द महिलाओं (शाहबानों, सायरा बानो, इशरत जहाँ और जकिय सोनम आदि) के जागरूक होने से समाज की तस्वीर नही बदली जा सकती सभी महिलाओं को इसके लिए आगे आना होगा।

\section{SOURCES OF FUNDING}

None.

\section{CONFLICT OF INTEREST}

None.

\section{ACKNOWLEDGMENT}

None.

\section{REFERENCES}

[1] डॉ. चेतन मेहता "महिला एवं कानून" आशीष पब्लिशिंग हाऊस $8 / 81$ पंजाबी बाग, नई दिल्ली, 2004।

[2] विकिपीडिया।

[3] पंजाब केसरी- 25 अगस्त 2017 (दिल्ली संस्करण)

[4] दैनिक जागरण- 23 अगस्त 2017 (इलाहाबाद संस्करण)

[5] हिन्दुस्तान- 25 अगस्त 2017 (लखनऊ, इलाहाबाद संस्करण)

[6] जनसत्ता- 26 अगस्त 2017 (लखनऊ, संस्करण)

[7] भारत में स्ती असमानताः- एक विमर्श, डा. गोया जोशी हिंदी माध्यम कार्यान्वय निदेशालय दिल्ली विश्वविद्यालय दिल्ली-110007

[8] Zakia A. Siddiki Anwar Jahan Jurveri Muslim Women Problems and Prospects New Delhi MD Pub. Pvt. Ltd

[9] विनायक त्रिपाटी:- भारतीय संस्कृति में मानवाधिकार उत्पत्ति एवं अवधारणा ओमेगा पब्लिकेशन नई दिल्ली-110002

[10] उपर्युक्त

[11] जनसत्ता-लखनऊ संस्करण 24 अगस्त 2017

[12] हिन्दुस्तान-24 अगस्त (इलाहाबाद संस्करण) 
[13] द हिन्दू-लखनऊ संस्करण 25 अगस्त 2017

[14] 20 सितम्बर 2018 जनसत्ता

[15] 02 अगस्त 2019 दैनिक जागरण। 